\title{
INSIDE LAB INVEST
}

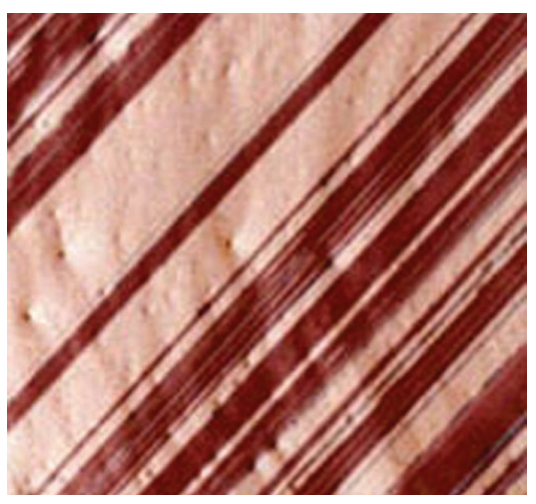

\section{Congo red-stained amyloid: not all apple green}

See page 232

Many pathologists loathe the thought of ordering a Congo red stain to identify amyloid in routine tissue sections. For some of us, the challenge lies in determining what hue really represents significant "apple green" among a spectrum of colors that frequently includes nonspecific autofluorescence. This is true whether controls or diagnostic samples are being evaluated. For others, the problem is the sometimes onerous search to find functional polarizers, which often disappear if not attached to the microscope. Regardless of how pathologists view this very common diagnostic method, most of us do not have a complete understanding of how it works, and this may add to our general disdain of the method.

Help is on the way. In this issue, Dr. Howie and colleagues provide an erudite and lucid explanation of the physical basis for colors observed in Congo-red-stained tissue sections under polarizing illumination. The carefully written, very informative report has enough background and definitions that the reader doesn't need to be a rocket scientist to fully understand it. The authors report that the spectrum of colors (not just apple green) produced by Congo red staining under polarization microscopy result from birefringent as well as absorptive/dichroic effects. The article provides a complete understanding of an optical phenomenon that is quite familiar to most pathologists but one that is not widely understood.

\section{Soluble factors from hypoxic placenta responsible for preeclampsia} See page 293

Preeclampsia is a relatively common pregnancy disorder that originates in the placenta and causes maternal and fetal problems. Hypoxic and dysfunctional placenta due to poor placentation is thought to release factors into the maternal circulation that cause the clinical features of preeclampsia. Although several candidate factors have been suggested, including vascular endothelial growth factor, none has yet been proved to be causative in vivo. Using placental villous explant culture, Robinson et al demonstrated that secreted factors from hypoxic placental tissue can alter multiple levels of endothelial cell function and hence mimic certain symptoms and biochemical alterations known to exist in preeclampsia. The ex vivo placental culture

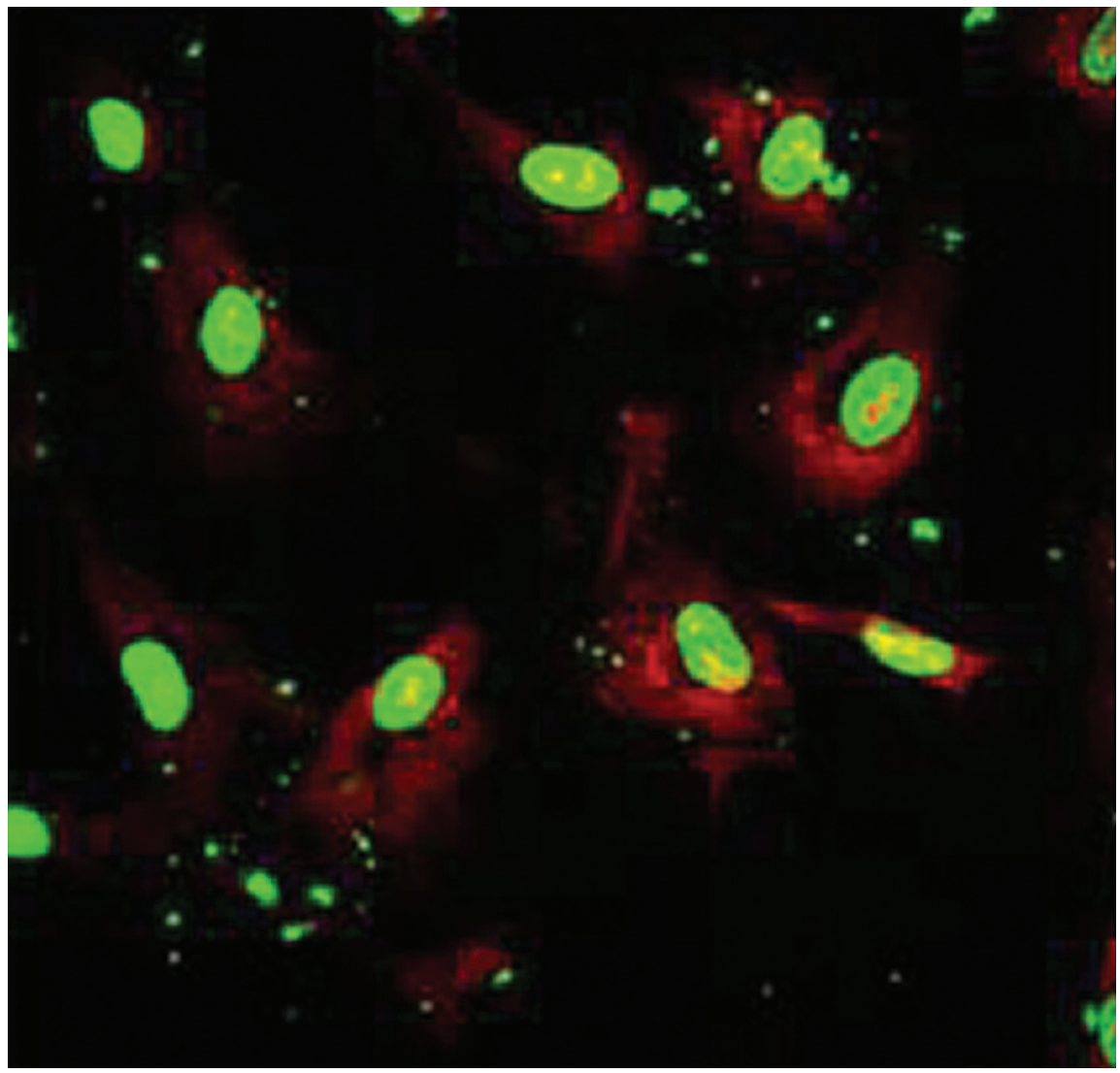




\section{nature.com/pathology}

system characterized in the present study appears to be promising in the future identification of a causative factor for preeclampsia.

\section{SHP-1 and inflammatory demyelination in multiple sclerosis}

See page 243

Multiple sclerosis (MS) is a disease of myelin loss and progressive degradation of central nervous system (CNS) function. Previous studies have shown that SHP-1deficient ("motheaten") mice develop a myelin deficiency and, when infected with Theiler's murine encephalomyelitis virus, CNS inflammatory demyelination. In this issue, Christophi et al report a relative deficiency in expression of the protein tyrosine phosphatase SHP-1 in lymphocytes from MS patients, compared with normal controls. Interestingly, this SHP-1 deficiency in MS patients correlates with a corresponding altered pattern of expression of genes regulated by STAT6, which is known to be heavily influenced by SHP-1 activity. Increasing SHP-1 expression in lymphocytes from MS patients with a lentiviral vector was sufficient to restore normal levels of STAT6 phosphorylation, whereas decreasing SHP-1 expression with siRNA in lymphocytes from normal controls resulted in MS-like levels of pSTAT6. These results establish SHP-1 as a key player in MS inflammation. The authors then demonstrate that the observed deficiency in promoter II SHP-1 transcripts in MS patients is due to epigenetic modifications. These observations in human samples may be of critical value in exploring mechanistic, diagnostic, and therapeutic options in MS.

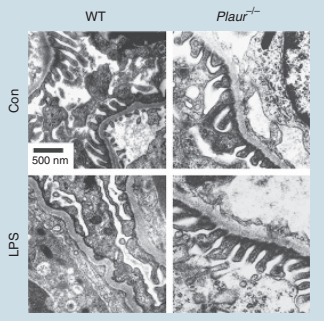

Function follows form? Proteinuria is a characteristic of inherited and acquired glomerular disease. Progress in understanding the more common acquired proteinuria syndromes has been slower than advances in hereditary proteinuria, but displacement of podocyte foot processes is recognized as a central factor. A recent study in Nature Medicine characterized the role of urokinase receptor (UPAR) expression on podocytes during development of acquired proteinuria. The work shows that UPAR expression is increased in podocytes of patients and experimental animals with proteinuric glomerular disease. More strikingly, UPAR knockout protects mice from experimental proteinuria. Further experiments demonstrate that UPAR activates integrins and promotes cell motility, explaining, in part, the basis of foot process effacement in proteinuria and the mechanisms that trigger development of this diagnostic morphology.

Nature Medicine 2008;14:55-63; doi:10.1038/nm1696

The immune system plays a new tune: baso novo Recent years have seen great progress in understanding the generation of $T$ helper types 1 and $17\left(T_{H} 1\right.$ and $\left.T_{H} 17\right) T$ cells in response to bacterial, viral, and fungal pathogens. In contrast, the control of $\mathrm{T}_{\mathrm{H}} 2 \mathrm{~T}$-cell differentiation, which occurs after helminth infection and in allergic responses, is less well characterized. A recent study in Nature Immunology demonstrates that basophils are activated and recruited to draining lymph nodes in response to $T_{H}$ 2-inducing allergens. Basophil migration is essential, as $T_{H}{ }^{2}$ responses are abolished in basophil-depleted mice. Consistent with a central role, allergen-activated basophils were shown to produce $\mathrm{T}_{\mathrm{H}} 2$-inducing cytokines. These data demonstrate the essential role of basophils in polarization of the innate immune response. Nature Immunology, published online 21 December 2007; doi:10.1038/ni1558

New recipe for multinucleated giant cells Mycobacterial infection, Crohn's disease, and rheumatoid arthritis share both the morphological characteristic of multinucleated giant cells and the biochemical characteristic of elevated interleukin-17 (IL-17). A recent study of patients with Langerhans cell histiocytosis ( $(\mathrm{CH})$ showed high serum IL-17A levels during active disease due to IL-17A synthesis by dendritic cells (DCs). Further analysis showed that IL-17A could induce in vitro fusion of monocytederived DCs from $\mathrm{LCH}$ patients as well as healthy donors. This DC fusion

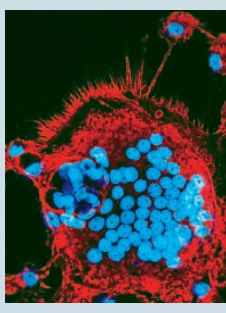
was enhanced by the addition of interferon- $\gamma$. These data suggest that IL-17A is critical to the development of multinucleated giant cells in LCH and, indirectly, implicate IL-17A in giant cell formation in mycobacterial infection, Crohn's disease, and rheumatoid arthritis. Whether IL-17A neutralization can help in the management of these diseases remains to be seen.

Nature Medicine 2008; 14:55-63; doi:10.1038/nm1694

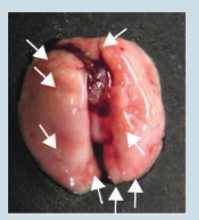

A functional biomarker for invasive ductal carcinoma? Epidermal growth factor receptor (EGFR) plays key roles in tumor invasion and metastasis. Identification of EGFR signaling pathways used in tumors but not by benign epithelia could lead to novel therapeutic approaches, but these pathways are poorly understood. One clue may be Arf6, a small GTPase overexpressed in highly invasive breast cancer cells that is only weakly expressed in noninvasive cancer and normal mammary epithelium. A recent letter in Nature Cell Biology has identified the guanine nucleotide exchange factor GEP100/BRAG2 as a critical component of the mechanism by which Arf6 triggers tumor invasion. The results show that GEP100 links EGFR signaling to Arf6 activation and suggest that GEP100 expression may be an important biomarker

of aggressive behavior in ductal breast cancer.

Nature Cell Biology 2008;10:85-92; doi:10.1038/ncb1672 